SAND2004-XXXX 2487

Printed XXXX 2004 June 2004

\title{
Critical Time Step for a Bilinear Laminated Composite Mindlin Shell Element
}

\author{
Daniel C. Hammerand \\ Material Mechanics \\ Sandia National Laboratories \\ P.O. Box 5800 \\ Albuquerque, NM 87185-0893
}

\begin{abstract}
The critical time step needed for explicit time integration of laminated shell finite element models is presented. Each layer is restricted to be orthotropic when viewed from a properly oriented material coordinate system. Mindlin shell theory is used in determining the laminated response that includes the effects of transverse shear. The effects of the membrane-bending coupling matrix from the laminate material model are included. Such a coupling matrix arises even in the case of non-symmetric lay-ups of differing isotropic layers. Single point integration is assumed to be used in determining a uniform strain response from the element. Using a technique based upon one from the literature, reduced eigenvalue problems are established to determine the remaining non-zero frequencies. It is shown that the eigenvalue problem arising from the inplane normal and shear stresses is decoupled from that arising from the transverse shear stresses. A verification example is presented where the exact and approximate results are compared.
\end{abstract}




\section{Acknowledgments}

David Day is thanked for the several discussions on alternative ways to estimate eigenvalues. The author also gratefully acknowledges the many helpful comments and suggestions provided by the reviewers Bob Chambers and Sam Key. 


\section{Contents}

$\begin{array}{lll}1 & \text { Introduction } & 7\end{array}$

2 Critical Time Step and General Eigenvalue Problem $\quad 8$

3 Displacements and Linear Strains 10

4 Internal Force Vector $\quad 12$

5 Midpoint Gradient Operators $\quad 15$

6 Constitutive Model 16

$\begin{array}{lll}7 & \text { Reduced Eigenvalue Problem } & 18\end{array}$

8 Critical Time Step Estimate $\quad 21$

9 Single Layer Reductions $\quad 23$

9.1 General Single Layer . . . . . . . . . . . . . . . . . . . . . . 23

9.2 Single Specially Orthotropic Layer . . . . . . . . . . . . . 25

9.3 Single Isotropic Layer . . . . . . . . . . . . . . . . 26

9.3.1 Membrane and Bending Behaviors . . . . . . . . . . . 26

9.3.2 Transverse Shear Behavior . . . . . . . . . . . . . . . 28

9.3.3 Significance of Single Isotropic Layer Criteria . . . . . . . . . . 30

$\begin{array}{ll}10 \text { Verification Problem } & 31\end{array}$

$\begin{array}{ll}11 \text { Summary } & 34\end{array}$

$\begin{array}{ll}\text { References } & 35\end{array}$

\section{List of Figures}

10.1 Example element geometry and coordinate systems. . . . . . . . . . 32 


\section{List of Tables}

10.1 Nodal coordinates for single element example. . . . . . . . . . . . . . . 31

10.2 Results for $\Delta t_{c r}$ for membrane/bending and transverse shear waves. . 33 


\section{Introduction}

Composite materials are of growing importance to Sandia National Laboratories. The composite structures of interest can be analyzed using nonlinear quasi-statics (ADAGIO), implicit structural dynamics (SALINAS), and explicit dynamics (PRESTO) finite element codes. One issue specific for explicit dynamics is the calculation of a stable time step for the conditionally stable central difference time integrator. ${ }^{1}$ This stable time step is typically estimated using a bound calculated from examining each element individually. Furthermore, this estimate for each element is usually found by applying a CFL (Courant, Friedrichs, Lewy) type of approach where the critical time step is found as the time for a wave to cross a characteristic element dimension. ${ }^{2,3}$ In fact, this is what is currently used in PRESTO for most cases. However, the response of a laminated composite is anisotropic and requires that the directional nature of the response be taken into account.

This report details the derivation of membrane/bending and transverse shear eigenvalue problems leading to bounds for the critical time step of 4-noded Mindlin shell elements using the elastic_laminate model of PRESTO. The elastic_laminate constitutive model for shells has been detailed in Ref. 4. PRESTO uses single point integration at the element centroid in determining the element internal force. Hence, in addition to the zero eigenvalues arising from rigid body modes, zero eigenvalues also result from the hourglass modes created from using single point integration. Using a methodology similar to that given in Refs. 3 and 5 , all of the zero energy modes are eliminated from consideration in the developed eigenvalue problems. In the current formulation, the one to one correspondence between the reduced problem size and the number of independent uniform force and force-couple quantities for the underintegrated element is explicitly delineated. The reduced problem size allows for simpler and more precise calculations/estimates to be used in determining the critical eigenvalues required for computing the critical time step. Simplifications of the laminate eigenvalue problems appropriate for various classes of single layer laminates are given. Also given are methods to quickly estimate the critical time step for membrane/bending waves. Finally, an example problem from the PRESTO regression test suite is detailed. 


\section{Critical Time Step and General Eigenvalue Problem}

It is well-known that the critical time step for central difference method when applied to linear finite element analysis is determined as ${ }^{1}$

$$
\Delta t_{c r}=\frac{2}{\omega_{\max }}
$$

where $\omega_{\max }$ is the maximum eigenvalue determined from the free vibration of the assembled finite element system. That is, $\omega_{\max }$ is determined from considering

$$
\left|[K]-\omega^{2}[M]\right|=0
$$

where $[K]$ and $[M]$ are the assembled stiffness and mass matrices. For nonlinear analysis, the upper limit on the time step necessary to prevent instability is taken to be equal to that computed from Eqs. (2.1) and (2.2) using $[K]$ and $[M]$ evaluated at the start of the time step in question. It can be shown that ${ }^{1}$

$$
\omega_{\max } \leq \max _{(n)} \omega_{\max }^{(n)}
$$

where $\omega_{\max }^{(n)}$ is the maximum frequency of the $n^{\text {th }}$ element. Hence, in the development to follow, the critical time step will be developed by considering a single element and using Eq. (2.3) which is equivalent to using Eq. (2.2) for a uniform mesh of identical elements.

The eigenvalue problem for a single element is determined starting from the spatiallydiscretized equations of motion for free vibration which are written as follows:

$$
[m]\{\ddot{d}\}+[k]\{d\}=\{0\}
$$

where $[m]$ and $[k]$ are the element mass and stiffness matrices, $\{d\}$ is the displacement vector of nodal translational and rotational degrees-of-freedom (DOF), and $\{\ddot{d}\}$ denotes $\partial^{2}\{d\} / \partial t^{2}$, the corresponding vector of accelerations. The eigenvalue problem is derived by replacing the vector of nodal DOF by the following expression:

$$
\{d\}=\{\chi\} e^{i \omega t}
$$

where $\{\chi\}$ is the vector of displacement amplitudes and $\omega$ now denotes the free vibration frequencies for a single element. After substituting Eq. (2.5) into Eq. (2.4), the 
time dependence is eliminated by dividing both sides by $e^{i \omega t}$ to give the eigenvalue problem as

$$
[k]\{\chi\}=\omega^{2}[m]\{\chi\}
$$

An equivalent statement of the eigenvalue problem is determined by multiplying both sides of Eq. (2.6) by $[m]^{-1}$ to give

$$
[m]^{-1}[k]\{\chi\}=\omega^{2}\{\chi\}
$$

In order to avoid having to solve a system of equations at each time step, a lumped mass matrix is used in the central difference method to turn it into an explicit method. The vector $\{d\}$ is written with the rotational DOF at its end so that

$$
[m]=\frac{\rho A h}{4}\left[\begin{array}{cc}
{\left[I_{(12 \times 12)}\right]} & {[0]} \\
{[0]} & \alpha\left[I_{(8 x 8)}\right]
\end{array}\right]
$$

where $\rho$ is the element density, $A$ is the element area, $h$ is the element thickness, $\alpha$ is the rotational inertia scaling factor, and $\left[I_{(N \times N)}\right]$ is the $(N \times N)$ identity matrix. One choice for $\alpha$ is ${ }^{6}$

$$
\alpha=\frac{A}{12}
$$

which when multiplied by $\rho A h / 4$ gives the mass moment of inertia of one-fourth of a rigid square element. Another choice is to set $\alpha$ to be the ratio of the area moment of inertia $I$ to the area $A$ as follows: ${ }^{7}$

$$
\alpha=\frac{I}{A}=\frac{h^{2}}{12}
$$

PRESTO uses Eq. (2.9) in its internal computations. The lumped mass matrix is rewritten as

$$
[m]=\frac{\rho A h \alpha}{4}\left[\begin{array}{cc}
\frac{1}{\alpha}\left[I_{(12 \times 12)}\right] & {[0]} \\
{[0]} & {\left[I_{(8 x 8)}\right]}
\end{array}\right]=\frac{\rho A h \alpha}{4}[\hat{m}]
$$

Furthermore, $[k]\{\chi\}$ is recognized as the amplitude vector corresponding to the element internal force vector so that Eq. (2.6) is rewritten as

$$
\frac{\rho A h \alpha}{4} \omega^{2}[\hat{m}]\{\chi\}=\left\{f_{\text {int }}\right\}
$$

For non-zero frequencies, this equation can be rearranged further to give the displacement amplitude vector in the eigenvalue problem written as

$$
\{\chi\}=\frac{4}{\rho A h \alpha \omega^{2}}[\hat{m}]^{-1}\left\{f_{\text {int }}\right\}
$$


Although many details are to follow, the reduced eigenvalue problem will be developed by writing the amplitude vectors of the laminate force and force-couple resultants (stress and stress times moment arm integrated through the thickness) in terms of $\{\chi\}$ and then using Eq. (2.13) to express $\{\chi\}$ in terms of $\left\{f_{\text {int }}\right\}$ which is in turn written in terms of the amplitude vectors of the force and force-couple resultants. The end result will be an eigenvalue problem where linear combinations of force and forcecouple resultant amplitudes are expressed in terms of a scalar (an eigenvalue) times the force and force-couple amplitudes themselves. From this point forward, the distinction between actual oscillating quantities and their associated amplitude vectors will not be explicitly made. Rather, the difference should be clear from the context in which the quantities appear.

The expression for the internal force vector will be derived using the Principle of Virtual Work. That is, $\left\{f_{\text {int }}\right\}$ is determined from the internal virtual work $\delta W_{\text {int }}$ which is written as

$$
\delta W_{i n t}=\int_{V} \sigma_{i j} \delta \epsilon_{i j} d V=\{\delta d\}^{T}\left\{f_{i n t}\right\}
$$

where $\delta \epsilon_{i j}$ are the virtual small strains (only including linear terms), $\sigma_{i j}$ are the stresses, $V$ is the element volume, and repeated indices are summed. Hence, it is now necessary to relate the virtual strains to the virtual displacements.

\section{Displacements and Linear Strains}

The displacement field for the 4-node bilinear shell element is expressed as

$$
\left\{\begin{array}{c}
U(x, y, z) \\
V(x, y, z) \\
W(x, y, z)
\end{array}\right\}=\left\{\begin{array}{l}
u(x, y) \\
v(x, y) \\
w(x, y)
\end{array}\right\}+z\left\{\begin{array}{c}
\theta_{y}(x, y) \\
-\theta_{x}(x, y) \\
0
\end{array}\right\}
$$

where $U, V$, and $W$ are the displacements in the $x, y$, and $z$ directions, $u, v$, and $w$ are the corresponding midplane deflections, and $\theta_{x}$ and $\theta_{y}$ are the rotations about the $x$ and $y$ axes. Here $z$ has been defined to be the direction perpendicular to the shell. For simplicity, all notation throughout the theory portion of this report will use $x-y$ $z$ coordinate system in expressing directions and components. However, the results that are derived can be equally applied in another orthogonal coordinate system such 
as the $r$-s-t co-rotational coordinate system actually employed in internal PRESTO calculations. From this point forward, the shell element will be considered to be flat.

Considering only linear terms, the inplane strains are written as

$$
\left\{\begin{array}{c}
\epsilon_{x x} \\
\epsilon_{y y} \\
2 \epsilon_{x y}
\end{array}\right\}=\left\{\begin{array}{c}
\frac{\partial U}{\partial x} \\
\frac{\partial V}{\partial y} \\
\frac{\partial U}{\partial y}+\frac{\partial V}{\partial x}
\end{array}\right\}=\left\{\begin{array}{c}
\frac{\partial u}{\partial x} \\
\frac{\partial v}{\partial y} \\
\frac{\partial u}{\partial y}+\frac{\partial v}{\partial x}
\end{array}\right\}+z\left\{\begin{array}{c}
\frac{\partial \theta_{y}}{\partial x} \\
-\frac{\partial \theta_{x}}{\partial y} \\
\frac{\partial \theta_{y}}{\partial y}-\frac{\partial \theta_{x}}{\partial x}
\end{array}\right\}
$$

Letting $\{\epsilon\},\{e\}$, and $\{\kappa\}$ denote total inplane strains, midplane strains, and bending curvatures, respectively, Eq. (3.2) is rewritten as

$$
\{\epsilon\}=\{e\}+z\{\kappa\}
$$

The transverse shear strains are written as

$$
\left\{\begin{array}{c}
2 \epsilon_{y z} \\
2 \epsilon_{z x}
\end{array}\right\}=\left\{\begin{array}{c}
\frac{\partial W}{\partial y}+\frac{\partial V}{\partial z} \\
\frac{\partial W}{\partial x}+\frac{\partial U}{\partial z}
\end{array}\right\}=\left\{\begin{array}{l}
\frac{\partial w}{\partial y}-\theta_{x} \\
\frac{\partial w}{\partial x}+\theta_{y}
\end{array}\right\}
$$

The transverse shear strains are constant throughout the thickness and are written as

$$
\left\{\epsilon_{t s}\right\}=\left\{e_{t s}\right\}
$$

Let the nodal degrees-of-freedom be arranged in $\{d\}$ as follows:

$$
\{d\}=\left\{\begin{array}{c}
\{u\} \\
\{v\} \\
\{w\} \\
\left\{\theta_{x}\right\} \\
\left\{\theta_{y}\right\}
\end{array}\right\}
$$

where $\{u\},\{v\},\{w\},\left\{\theta_{x}\right\}$, and $\left\{\theta_{y}\right\}$ are vectors of nodal displacements and rotations. The bilinear displacement field is then expressed in terms of these quantities using the bilinear shape functions $\{\Phi\}$ as follows:

$$
\begin{gathered}
u(x, y)=\{\Phi(x, y)\}^{T}\{u\}, \quad v(x, y)=\{\Phi(x, y)\}^{T}\{v\}, \quad w(x, y)=\{\Phi(x, y)\}^{T}\{w\} \\
\theta_{x}(x, y)=\{\Phi(x, y)\}^{T}\left\{\theta_{x}\right\}, \quad \& \quad \theta_{y}(x, y)=\{\Phi(x, y)\}^{T}\left\{\theta_{y}\right\}
\end{gathered}
$$


The linear strain displacement matrices are then derived by substituting the expressions listed in Eq. (3.7) into Eqs. (3.2) and (3.4) to give

$$
\begin{aligned}
\{e\} & =\left[G_{1}\right]\{d\} \\
\{\kappa\} & =\left[G_{2}\right]\{d\} \\
\left\{e_{t s}\right\} & =\left[G_{3}\right]\{d\}
\end{aligned}
$$

where $\left[G_{1}\right]$ is a $(3 \times 20)$ matrix defined as

$$
\left[G_{1}\right]=\left[\begin{array}{ccccc}
\frac{\partial\{\Phi\}^{T}}{\partial x} & \{0\}^{T} & \{0\}^{T} & \{0\}^{T} & \{0\}^{T} \\
\{0\}^{T} & \frac{\partial\{\Phi\}^{T}}{\partial y} & \{0\}^{T} & \{0\}^{T} & \{0\}^{T} \\
\frac{\partial\{\Phi\}^{T}}{\partial y} & \frac{\partial\{\Phi\}^{T}}{\partial x} & \{0\}^{T} & \{0\}^{T} & \{0\}^{T}
\end{array}\right]
$$

$\left[G_{2}\right]$ is a $(3 \times 20)$ matrix defined as

$$
\left[G_{2}\right]=\left[\begin{array}{ccccc}
\{0\}^{T} & \{0\}^{T} & \{0\}^{T} & \{0\}^{T} & \frac{\partial\{\Phi\}^{T}}{\partial x} \\
\{0\}^{T} & \{0\}^{T} & \{0\}^{T} & -\frac{\partial\{\Phi\}^{T}}{\partial y} & \{0\}^{T} \\
\{0\}^{T} & \{0\}^{T} & \{0\}^{T} & -\frac{\partial\{\Phi\}^{T}}{\partial x} & \frac{\partial\{\Phi\}^{T}}{\partial y}
\end{array}\right]
$$

and $\left[G_{3}\right]$ is a $(2 \times 20)$ matrix defined as

$$
\left[G_{3}\right]=\left[\begin{array}{ccccc}
\{0\}^{T} & \{0\}^{T} & \frac{\partial\{\Phi\}^{T}}{\partial y} & -\{\Phi\}^{T} & \{0\}^{T} \\
\{0\}^{T} & \{0\}^{T} & \frac{\partial\{\Phi\}^{T}}{\partial x} & \{0\}^{T} & \{\Phi\}^{T}
\end{array}\right]
$$

\section{Internal Force Vector}

The virtual small strains are now written as

$$
\{\delta \epsilon\}=\{\delta e\}+z\{\delta \kappa\}
$$

and

$$
\left\{\delta \epsilon_{t s}\right\}=\left\{\delta e_{t s}\right\}
$$


Substituting Eqs. (4.1) and (4.2) into Eq. (2.14) yields

$$
\delta W_{\text {int }}=\int_{A} \int_{-h / 2}^{h / 2}\left(\{\delta e\}^{T}+z\{\delta \kappa\}^{T}\right)\{\sigma\} d z d A+\int_{A} \int_{-h / 2}^{h / 2}\left\{\delta e_{t s}\right\}^{T}\left\{\sigma_{t s}\right\} d z d A
$$

where $\{\sigma\}$ is the vector of inplane normal and shear stresses, $\left\{\sigma_{t s}\right\}$ is the vector of transverse shear stresses, and $h$ is the laminate thickness. However, it is known that modeling the transverse shear strains and stresses to be constant through the thickness results in the transverse shear energy being too large compared to that coming from a more realistic parabolic distribution. A transverse shear correction factor of $\beta^{2}=5 / 6$ can be used so that the constant distribution of transverse shear strains and stresses has the same strain energy as a parabolic distribution. ${ }^{8}$ Using such a transverse shear correction factor, the internal virtual work would become

$$
\delta W_{\text {int }}=\int_{A} \int_{-h / 2}^{h / 2}\left(\{\delta e\}^{T}+z\{\delta \kappa\}^{T}\right)\{\sigma\} d z d A+\beta^{2} \int_{A} \int_{-h / 2}^{h / 2}\left\{\delta e_{t s}\right\}^{T}\left\{\sigma_{t s}\right\} d z d A
$$

However, sometimes it is advantageous to use a different transverse shear correction factor. As the laminate thickness decreases to zero, Kirchhoff bending conditions of zero transverse shear strains should result. It is desired to achieve this behavior without shear locking the element. Based on the work presented in Ref. 9, the transverse shear correction factor can be used to recover this thin shell behavior. That is, the transverse shear correction factor becomes a penalty multiplier with the transverse shear energy acting as a penalty function for modeling thin shell behavior. Fried et al. ${ }^{9}$ proposed a transverse shear correction factor $\beta^{2}=6 h^{2} / A$ to achieve thin shell behavior without shear locking the element. Then the minimum of $\beta^{2}=5 / 6$ and $\beta^{2}=6 h^{2} / A$ would be used. However, in the present work, a separate transverse shear correction factor is used for the $y z$ and $z x$ transverse shears as follows:

$$
\begin{aligned}
& \beta_{y z}^{2}=\min \left\{5 / 6,6 h^{2} / L_{y z}^{2}\right\} \\
& \beta_{z x}^{2}=\min \left\{5 / 6,6 h^{2} / L_{z x}^{2}\right\}
\end{aligned}
$$

where $L_{y z}$ and $L_{z x}$ are characteristic lengths of the element in the $y$ - and $x$-directions, respectively.

Using separate transverse shear correction factors for the $y z$ and $z x$ transverse 
shears, the internal virtual work can be written as

$$
\delta W_{\text {int }}=\int_{A} \int_{-h / 2}^{h / 2}\left(\{\delta e\}^{T}+z\{\delta \kappa\}^{T}\right)\{\sigma\} d z d A+\int_{A} \int_{-h / 2}^{h / 2}\left\{\beta \delta e_{t s}\right\}^{T}\left\{\beta \sigma_{t s}\right\} d z d A
$$

where the vectors of scaled transverse shear strains and stresses are, respectively, given by

$$
\left\{\beta \delta e_{t s}\right\}=\left\{\begin{array}{c}
2 \beta_{y z} \delta e_{y z} \\
2 \beta_{z x} \delta e_{z x}
\end{array}\right\}
$$

and

$$
\left\{\beta \sigma_{t s}\right\}=\left\{\begin{array}{c}
\beta_{y z} \sigma_{y z} \\
\beta_{z x} \sigma_{z x}
\end{array}\right\}
$$

The internal force vector can then be determined using Eq. (2.14) as

$$
\left\{f_{\text {int }}\right\}=\int_{A}\left(\left[G_{1}\right]^{T}\{N\}+\left[G_{2}\right]^{T}\{M\}+\left[\beta G_{3}\right]^{T}\left\{\beta N_{t s}\right\}\right) d A
$$

where $\{N\}$ is the vector of inplane force resultants defined as

$$
\{N\}=\int_{-h / 2}^{h / 2}\{\sigma\} d z=\int_{-h / 2}^{h / 2}\left\{\begin{array}{c}
\sigma_{x x} \\
\sigma_{y y} \\
\sigma_{x y}
\end{array}\right\} d z
$$

$\{M\}$ is the vector of force-couple resultants defined as

$$
\{M\}=\int_{-h / 2}^{h / 2} z\{\sigma\} d z=\int_{-h / 2}^{h / 2} z\left\{\begin{array}{c}
\sigma_{x x} \\
\sigma_{y y} \\
\sigma_{x y}
\end{array}\right\} d z
$$

$\left\{\beta N_{t s}\right\}$ is the vector of scaled transverse shear force resultants defined as

$$
\left\{\beta N_{t s}\right\}=\int_{-h / 2}^{h / 2}\left\{\beta \sigma_{t s}\right\} d z=\int_{-h / 2}^{h / 2}\left\{\begin{array}{c}
\beta_{y z} \sigma_{y z} \\
\beta_{z x} \sigma_{z x}
\end{array}\right\} d z
$$

and $\left[\beta G_{3}\right]$ is given by

$$
\left[\beta G_{3}\right]=\left[\begin{array}{ccccc}
\{0\}^{T} & \{0\}^{T} & \beta_{y z} \frac{\partial\{\Phi\}^{T}}{\partial y} & -\beta_{y z}\{\Phi\}^{T} & \{0\}^{T} \\
\{0\}^{T} & \{0\}^{T} & \beta_{z x} \frac{\partial\{\Phi\}^{T}}{\partial x} & \{0\}^{T} & \beta_{z x}\{\Phi\}^{T}
\end{array}\right]
$$


The square root of the transverse shear correction factor was applied to the transverse shear stresses and the virtual transverse shear strains in Eq. (4.7) so that using a linear elastic material law will allow the transverse shear part of the problem to remain selfadjoint. That is, the gradient operator $\left[\beta G_{3}\right]$ used to compute the scaled transverse shear strains which are used in computing the scaled transverse shear stresses remains equal to the transpose of the divergence operator $\left[\beta G_{3}\right]^{T}$ which is used to compute the scaled transverse shear internal forces from the scaled transverse shear stresses via Eq. (4.10). ${ }^{10}$

The size of the free vibration eigenvalue problem arising from Eq. (2.13) is reduced when a single-point integration scheme is applied to Eq. (4.10). ${ }^{3,5}$ Letting the superscript 0 denote quantities evaluated at the element centroid, the internal force vector using single point integration is written as

$$
\left\{f_{\text {int }}^{0}\right\}=A\left(\left[G_{1}^{0}\right]^{T}\left\{N^{0}\right\}+\left[G_{2}^{0}\right]^{T}\left\{M^{0}\right\}+\left[\beta G_{3}^{0}\right]^{T}\left\{\beta N_{t s}^{0}\right\}\right)
$$

\section{Midpoint Gradient Operators}

The spatial derivatives of the element shape functions evaluated at the element centroid will be expressed in terms of $\left\{b_{1}\right\},\left\{b_{2}\right\}$, and $\left\{b_{3}\right\}$ which are defined as follows:

$$
\begin{aligned}
& \left\{b_{1}\right\}=A\left(\frac{\partial\{\Phi\}}{\partial x}\right)^{0} \\
& \left\{b_{2}\right\}=A\left(\frac{\partial\{\Phi\}}{\partial y}\right)^{0} \\
& \left\{b_{3}\right\}=A\left\{\Phi^{0}\right\}
\end{aligned}
$$

It can be shown in a straightforward manner that

$$
\begin{aligned}
\left\{b_{1}\right\}^{T} & =\frac{1}{2}\left\{\begin{array}{llll}
y_{24} & y_{31} & y_{42} & y_{13}
\end{array}\right\} \\
\left\{b_{2}\right\}^{T} & =\frac{1}{2}\left\{\begin{array}{llll}
x_{42} & x_{13} & x_{24} & x_{31}
\end{array}\right\} \\
\left\{b_{3}\right\}^{T} & =\frac{A}{4}\left\{\begin{array}{llll}
1 & 1 & 1 & 1
\end{array}\right\} \\
A & =\frac{1}{2}\left(x_{31} y_{42}+x_{24} y_{31}\right)
\end{aligned}
$$


where $x_{i j}$ is determined in terms of the nodal $x_{i}$ as

$$
x_{i j}=x_{i}-x_{j}
$$

Similar equations hold for $y_{i j}$.

The linear strain-displacement matrices evaluated at the element centroid are now written as

$$
\begin{gathered}
{\left[G_{1}^{0}\right]=\frac{1}{A}\left[\begin{array}{ccccc}
\left\{b_{1}\right\}^{T} & \{0\}^{T} & \{0\}^{T} & \{0\}^{T} & \{0\}^{T} \\
\{0\}^{T} & \left\{b_{2}\right\}^{T} & \{0\}^{T} & \{0\}^{T} & \{0\}^{T} \\
\left\{b_{2}\right\}^{T} & \left\{b_{1}\right\}^{T} & \{0\}^{T} & \{0\}^{T} & \{0\}^{T}
\end{array}\right]} \\
{\left[G_{2}^{0}\right]=\frac{1}{A}\left[\begin{array}{ccccc}
\{0\}^{T} & \{0\}^{T} & \{0\}^{T} & \{0\}^{T} & \left\{b_{1}\right\}^{T} \\
\{0\}^{T} & \{0\}^{T} & \{0\}^{T} & -\left\{b_{2}\right\}^{T} & \{0\}^{T} \\
\{0\}^{T} & \{0\}^{T} & \{0\}^{T} & -\left\{b_{1}\right\}^{T} & \left\{b_{2}\right\}^{T}
\end{array}\right]} \\
{\left[\beta G_{3}^{0}\right]=\frac{1}{A}\left[\begin{array}{ccccc}
\{0\}^{T} & \{0\}^{T} & \beta_{y z}\left\{b_{2}\right\}^{T} & -\beta_{y z}\left\{b_{3}\right\}^{T} & \{0\}^{T} \\
\{0\}^{T} & \{0\}^{T} & \beta_{z x}\left\{b_{1}\right\}^{T} & \{0\}^{T} & \beta_{z x}\left\{b_{3}\right\}^{T}
\end{array}\right]}
\end{gathered}
$$

In the development to follow it will be useful to define the dot products of these vectors as follows:

$$
a_{i j}=\left\{b_{i}\right\}^{T}\left\{b_{j}\right\}
$$

Using Eqs. (5.4)-(5.6), these geometric factors are written explicitly in terms of $x_{i j}$, $y_{i j}$, and $A$ as

$$
\left[\begin{array}{ccc}
a_{11} & a_{12} & a_{13} \\
a_{21} & a_{22} & a_{23} \\
a_{31} & a_{32} & a_{33}
\end{array}\right]=\left[\begin{array}{ccc}
\frac{1}{2}\left(y_{24}^{2}+y_{31}^{2}\right) & \frac{1}{2}\left(y_{24} x_{42}+y_{31} x_{13}\right) & 0 \\
& \frac{1}{2}\left(x_{24}^{2}+x_{31}^{2}\right) & 0 \\
S Y M & & \frac{A^{2}}{4}
\end{array}\right]
$$

\section{Constitutive Model}

The material response is modeled using a linear elastic anisotropic constitutive equation as described for the elastic_laminate model in Ref. 4. Such models are typically used in representing the response of fiber-reinforced laminated composites. Note that in Ref. 4, the stresses arising from thermal changes have been included. However, 
these loads can be considered as applied external loads and need not be included here for determining the critical time step. Generalized plane stress conditions will be used such that the constitutive equation for inplane stresses at a given $z$ location through the thickness is

$$
\left\{\begin{array}{c}
\sigma_{x x} \\
\sigma_{y y} \\
\sigma_{x y}
\end{array}\right\}=\left[\begin{array}{lll}
\bar{Q}_{11} & \bar{Q}_{12} & \bar{Q}_{16} \\
\bar{Q}_{12} & \bar{Q}_{22} & \bar{Q}_{26} \\
\bar{Q}_{16} & \bar{Q}_{26} & \bar{Q}_{66}
\end{array}\right]\left\{\left\{\begin{array}{c}
e_{x x} \\
e_{y y} \\
2 e_{x y}
\end{array}\right\}+z\left\{\begin{array}{c}
\kappa_{x x} \\
\kappa_{y y} \\
2 \kappa_{x y}
\end{array}\right\}\right\}
$$

and that for the scaled transverse shear stresses is

$$
\left\{\begin{array}{c}
\beta_{y z} \sigma_{y z} \\
\beta_{z x} \sigma_{z x}
\end{array}\right\}=\left[\begin{array}{ll}
\bar{Q}_{44} & \bar{Q}_{45} \\
\bar{Q}_{45} & \bar{Q}_{55}
\end{array}\right]\left\{\begin{array}{l}
2 \beta_{y z} e_{y z} \\
2 \beta_{z x} e_{z x}
\end{array}\right\}
$$

The form of the material model given by Eqs. (6.1) and (6.2) can be taken to correspond to a truly anisotropic material with no coupling between inplane normal/shear and transverse shear behavior, or to one which is truly orthotropic when viewed in a coordinate system aligned with material directions. That is, letting the material coordinate system be denoted by 1-2-3, the constitutive model can be expressed as

$$
\left\{\begin{array}{c}
\sigma_{11} \\
\sigma_{22} \\
\beta_{23} \sigma_{23} \\
\beta_{31} \sigma_{31} \\
\sigma_{12}
\end{array}\right\}=\left[\begin{array}{ccccc}
Q_{11} & Q_{12} & 0 & 0 & 0 \\
Q_{12} & Q_{22} & 0 & 0 & 0 \\
0 & 0 & Q_{44} & 0 & 0 \\
0 & 0 & 0 & Q_{55} & 0 \\
0 & 0 & 0 & 0 & Q_{66}
\end{array}\right]\left\{\left\{\begin{array}{c}
e_{11} \\
e_{22} \\
2 \beta_{23} e_{23} \\
2 \beta_{31} e_{31} \\
2 e_{12}
\end{array}\right\}+z\left\{\begin{array}{c}
\kappa_{11} \\
\kappa_{22} \\
0 \\
0 \\
2 \kappa_{12}
\end{array}\right\}\right.
$$

Using standard tensor transformations, all the $\bar{Q}_{i j}$ 's can be expressed in terms of the $Q_{i j}$ 's.

Substituting Eq. (6.1) into Eqs. (4.11) and (4.12) gives the inplane force and forcecouple resultants as

$$
\left\{\begin{array}{l}
N_{x x} \\
N_{y y} \\
N_{x y} \\
M_{x x} \\
M_{y y} \\
M_{x y}
\end{array}\right\}=\left[\begin{array}{ccc|ccc}
A_{11} & A_{12} & A_{16} & B_{11} & B_{12} & B_{16} \\
A_{12} & A_{22} & A_{26} & B_{12} & B_{22} & B_{26} \\
A_{16} & A_{26} & A_{66} & B_{16} & B_{26} & B_{66} \\
\hline B_{11} & B_{12} & B_{16} & D_{11} & D_{12} & D_{16} \\
B_{12} & B_{22} & B_{26} & D_{12} & D_{22} & D_{26} \\
B_{16} & B_{26} & B_{66} & D_{16} & D_{26} & D_{66}
\end{array}\right]\left\{\begin{array}{c}
e_{x x} \\
e_{y y} \\
2 e_{x y} \\
\kappa_{x x} \\
\kappa_{y y} \\
2 \kappa_{x y}
\end{array}\right\}
$$


or

$$
\left\{\begin{array}{c}
\{N\} \\
\{M\}
\end{array}\right\}=\left[\begin{array}{cc}
{[A]} & {[B]} \\
{[B]} & {[D]}
\end{array}\right]\left\{\begin{array}{c}
\{e\} \\
\{\kappa\}
\end{array}\right\}
$$

where

$$
\left(A_{i j} ; B_{i j} ; D_{i j}\right)=\int_{-h / 2}^{h / 2}\left(1 ; z ; z^{2}\right) \bar{Q}_{i j} d z
$$

Likewise, integrating Eq. (6.2) through the thickness gives

$$
\left\{\begin{array}{c}
\beta_{y z} N_{y z} \\
\beta_{z x} N_{z x}
\end{array}\right\}=\left[\begin{array}{cc}
A_{44} & A_{45} \\
A_{45} & A_{55}
\end{array}\right]\left\{\begin{array}{l}
2 \beta_{y z} e_{y z} \\
2 \beta_{z x} e_{z x}
\end{array}\right\}
$$

or

$$
\left\{\beta N_{t s}\right\}=\left[A_{t s}\right]\left\{\beta e_{t s}\right\}
$$

Using Eqs. (3.8) and (3.9), the inplane force and force-couple resultants evaluated at the element centroid are

$$
\left\{N^{0}\right\}=[A]\left[G_{1}^{0}\right]\{d\}+[B]\left[G_{2}^{0}\right]\{d\}
$$

and

$$
\left\{M^{0}\right\}=[B]\left[G_{1}^{0}\right]\{d\}+[D]\left[G_{2}^{0}\right]\{d\}
$$

Likewise using Eq. (3.10), the scaled transverse shear force resultant evaluated at the centroid is

$$
\left\{\beta N_{t s}^{0}\right\}=\left[A_{t s}\right]\left[\beta G_{3}^{0}\right]\{d\}
$$

\section{Reduced Eigenvalue Problem}

For the eigenvalue problem, $\{d\}$ is replaced by $\{\chi\}$ in Eqs. (6.9)-(6.11) such that $\left\{N^{0}\right\},\left\{M^{0}\right\}$, and $\left\{N_{t s}^{0}\right\}$ refer specifically to the amplitude of the corresponding force or force-couple resultant. Then, $\{\chi\}$ is in turn rewritten in terms of $\left\{N^{0}\right\},\left\{M^{0}\right\}$, and $\left\{N_{t s}^{0}\right\}$ using Eqs. (2.13) and (4.15). The eigenvalue problem is then expressed by 
the following equations

$$
\begin{aligned}
\left\{N^{0}\right\}= & \frac{4}{\rho h \alpha \omega^{2}}\left(\left([A]\left[G_{1}^{0}\right][\hat{m}]^{-1}\left[G_{1}^{0}\right]^{T}+[B]\left[G_{2}^{0}\right][\hat{m}]^{-1}\left[G_{1}^{0}\right]^{T}\right)\left\{N^{0}\right\}\right. \\
& +\quad\left([A]\left[G_{1}^{0}\right][\hat{m}]^{-1}\left[G_{2}^{0}\right]^{T}+[B]\left[G_{2}^{0}\right][\hat{m}]^{-1}\left[G_{2}^{0}\right]^{T}\right)\left\{M^{0}\right\} \\
& \left.+\quad\left([A]\left[G_{1}^{0}\right][\hat{m}]^{-1}\left[\beta G_{3}^{0}\right]^{T}+[B]\left[G_{2}^{0}\right][\hat{m}]^{-1}\left[\beta G_{3}^{0}\right]^{T}\right)\left\{\beta N_{t s}^{0}\right\}\right) \\
\left\{M^{0}\right\}= & \frac{4}{\rho h \alpha \omega^{2}}\left(\left([B]\left[G_{1}^{0}\right][\hat{m}]^{-1}\left[G_{1}^{0}\right]^{T}+[D]\left[G_{2}^{0}\right][\hat{m}]^{-1}\left[G_{1}^{0}\right]^{T}\right)\left\{N^{0}\right\}\right. \\
& +\quad\left([B]\left[G_{1}^{0}\right][\hat{m}]^{-1}\left[G_{2}^{0}\right]^{T}+[D]\left[G_{2}^{0}\right][\hat{m}]^{-1}\left[G_{2}^{0}\right]^{T}\right)\left\{M^{0}\right\} \\
& \left.+\quad\left([B]\left[G_{1}^{0}\right][\hat{m}]^{-1}\left[\beta G_{3}^{0}\right]^{T}+[D]\left[G_{2}^{0}\right][\hat{m}]^{-1}\left[\beta G_{3}^{0}\right]^{T}\right)\left\{\beta N_{t s}^{0}\right\}\right) \\
\left\{\beta N_{t s}^{0}\right\}= & \frac{4}{\rho h \alpha \omega^{2}}\left(\left[A_{t s}^{0}\right]\left[\beta G_{3}^{0}\right][\hat{m}]^{-1}\left[G_{1}^{0}\right]^{T}\left\{N^{0}\right\}\right. \\
& +\quad\left[A_{t s}^{0}\right]\left[\beta G_{3}^{0}\right][\hat{m}]^{-1}\left[G_{2}^{0}\right]^{T}\left\{M^{0}\right\} \\
& \left.+\quad\left[A_{t s}^{0}\right]\left[\beta G_{3}^{0}\right][\hat{m}]^{-1}\left[\beta G_{3}^{0}\right]^{T}\left\{\beta N_{t s}^{0}\right\}\right)
\end{aligned}
$$

It can be shown that a large number of the terms in Eqs. (7.1)-(7.3) are zero. In fact,

$$
\left[G_{i}^{0}\right][\hat{m}]^{-1}\left[G_{j}^{0}\right]^{T}=0 \quad i \neq j
$$

even when the transverse shear correction factors are used to modify $\left[G_{3}^{0}\right]$. Hence, the eigenvalue problems arising from the inplane (normal and shear) stresses and transverse shear stresses are decoupled.

The following $(6 \times 6)$ eigenvalue problem results from the inplane stresses:

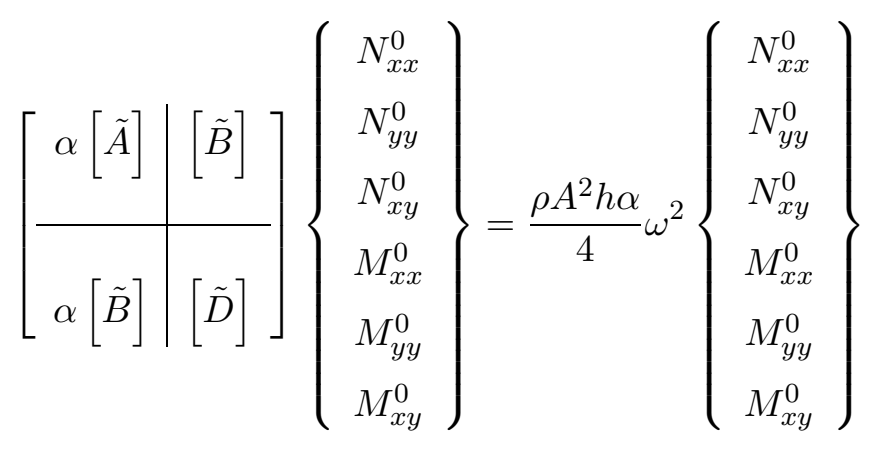


where

$$
\begin{aligned}
{[\tilde{A}] } & =\left[\begin{array}{ccc}
\tilde{A}_{11} & \tilde{A}_{12} & \tilde{A}_{16} \\
\tilde{A}_{21} & \tilde{A}_{22} & \tilde{A}_{26} \\
\tilde{A}_{61} & \tilde{A}_{62} & \tilde{A}_{66}
\end{array}\right] \\
& =\left[\begin{array}{ccc}
a_{11} A_{11}+a_{12} A_{16} & a_{22} A_{12}+a_{12} A_{16} & a_{12}\left(A_{11}+A_{12}\right)+\left(a_{11}+a_{22}\right) A_{16} \\
a_{11} A_{12}+a_{12} A_{26} & a_{22} A_{22}+a_{12} A_{26} & a_{12}\left(A_{12}+A_{22}\right)+\left(a_{11}+a_{22}\right) A_{26} \\
a_{11} A_{16}+a_{12} A_{66} & a_{22} A_{26}+a_{12} A_{66} & a_{12}\left(A_{16}+A_{26}\right)+\left(a_{11}+a_{22}\right) A_{66}
\end{array}\right]
\end{aligned}
$$

where the $a_{i j}$ 's are given in Eq. (5.13). Equations for $[\tilde{B}]$ and $[\tilde{D}]$ are obtained by replacing $A_{i j}$ by $B_{i j}$ and $D_{i j}$, respectively, in Eq. (7.6). Note that the $(6 \times 6)$ matrix in Eq. (7.5) is, in general, asymmetric. This asymmetry results from the gradient and divergence operators used in creating the reduced eigenvalue problem. This asymmetry remains in the case of symmetric lay-ups which necessarily have $[\tilde{B}]=[0]$ when $z=0$ for the element midplane, as chosen here. This, of course, includes the case of a single layer whether it be anisotropic or not. Furthermore, even considering the special sub-case of a single isotropic layer $\left(A_{11}=A_{22}, A_{12}=\nu A_{11}\right.$, $A_{16}=A_{26}=0, A_{66}=((1-\nu) / 2) A_{11} ; B_{11}=B_{22}=B_{12}=B_{16}=B_{26}=B_{66}=0 ;$ $\left.D_{11}=D_{22}=\left(h^{2} / 12\right) A_{11}, D_{12}=\nu D_{11}, D_{16}=D_{26}=0, D_{66}=((1-\nu) / 2) D_{11}\right)$, the eigenvalue problem given by Eq. (7.5) is asymmetric, unless the particular element under consideration is a perfect square $\left(a_{11}=a_{22}, a_{12}=0\right)$.

On the other hand, the following $(2 \times 2)$ eigenvalue problem results from the scaled transverse shear stresses:

$$
\left[\tilde{A}_{t s}\right]\left\{\begin{array}{c}
\beta_{y z} N_{y z}^{0} \\
\beta_{z x} N_{z x}^{0}
\end{array}\right\}=\frac{\rho A^{2} h \alpha}{4} \omega^{2}\left\{\begin{array}{c}
\beta_{y z} N_{y z}^{0} \\
\beta_{z x} N_{z x}^{0}
\end{array}\right\}
$$

where $\left[\tilde{A}_{t s}\right]$ is a $(2 \times 2)$ matrix given by

$$
\left[\tilde{A}_{t s}\right]=\left[\begin{array}{cc}
\tilde{A}_{44} & \tilde{A}_{45} \\
\tilde{A}_{54} & \tilde{A}_{55}
\end{array}\right]=\left[\begin{array}{c|c}
\left(\alpha a_{22}+a_{33}\right) \beta_{y z}^{2} A_{44} & \alpha \beta_{y z} \beta_{z x} a_{12} A_{44} \\
+\alpha \beta_{y z} \beta_{z x} a_{12} A_{45} & +\left(\alpha a_{11}+a_{33}\right) \beta_{z x}^{2} A_{45} \\
\hline\left(\alpha a_{22}+a_{33}\right) \beta_{y z}^{2} A_{45} & \alpha \beta_{y z} \beta_{z x} a_{12} A_{45} \\
+\alpha \beta_{y z} \beta_{z x} a_{12} A_{55} & +\left(\alpha a_{11}+a_{33}\right) \beta_{z x}^{2} A_{55}
\end{array}\right]
$$

If equal transverse shear factors are applied to both transverse shears $\left(\beta_{y z}=\beta_{z x}=\right.$ $\beta$ ), the transverse shear eigenvalue problem can be simplified by factoring $\beta^{2}$ out 
of $\left[\tilde{A}_{t s}\right]$. Nevertheless, the $(2 \times 2)$ matrix defining the transverse shear eigenvalue problem is generally asymmetric. However, unlike the membrane/bending eigenvalue problem, the matrix defining the transverse shear eigenvalues is symmetric for all element geometries when the laminate corresponds to a single isotropic layer.

Using a $x-y-z$ coordinate system with the $z$-axis parallel to the element normal requires only two rotational degrees-of-freedom at each node in addition to three translational degrees-of-freedom. Hence, with five degrees-of-freedom at four nodes, the eigenvalue problem cast in Eq. (2.6) specifies twenty eigenvalues including rigid body modes. Using the methodology presented in Refs. 3 and 5, single point integration has reduced the eigenvalue problem down to the computation of eight eigenvalues as specified by Eqs. (7.5) and (7.7). The reduction in problem size was accomplished by disregarding the rigid body and hourglass modes. Clearly the size of the reduced eigenvalue problems corresponds exactly to the number of independent force and forcecouple resultants that can be represented using single point integration. The maximum eigenvalue to be used in determining the final critical time step corresponding to a given element is the maximum of those obtained independently from Eqs. (7.5) and (7.7). It should be noted that the eigenvalue problem defined by Eq. (7.7) corresponds to propagation of a transverse shear wave across the element and not through its thickness.

\section{Critical Time Step Estimate}

For the case of transverse shear waves, the asymmetric $(2 \times 2)$ eigenvalue problem given in Eq. (7.7) is small enough to be solved exactly to give the maximum transverse shear frequency without incurring excessive computational cost. On the other hand, the asymmetric $(6 \times 6)$ membrane/eigenvalue problem stated in Eq. (7.5) can be expensive to solve for each element at each time step. Hence, several bounds for the maximum membrane/bending frequency will be developed.

One typical way to bound the maximum eigenvalue of a matrix is to use a Gerschgorin circle estimate. ${ }^{11}$ Following Ref. 11, Gerschgorin's theorem is given as 
Gerschgorin Circle Theorem Let $[A]$ be an $(n \times n)$ matrix and let $R_{i}$ denote the circle in the complex plane with center $A_{i i}$ and radius $\sum_{\substack{j=1 \\ j \neq i}}^{n}\left|A_{i j}\right|$; that is,

$$
R_{i}=\left\{z \in \mathcal{C}|| z-A_{i i}\left|\leq \sum_{\substack{j=1 \\ j \neq i}}^{n}\right| A_{i j} \mid\right\}
$$

where $\mathcal{C}$ is used to denote the complex plane. The eigenvalues of $[A]$ are contained within $R=\bigcup_{i=1}^{n} R_{i}$. Moreover, the union of any $k$ of these circles that do not intersect the remaining $(n-k)$ circles must contain precisely $k$ (counting multiplicities) of the eigenvalues.

For simplicity in notation, Eq. (7.5) is rewritten as follows:

$$
[P]\left\{\begin{array}{c}
N_{x x}^{0} \\
N_{y y}^{0} \\
N_{x y}^{0} \\
M_{x x}^{0} \\
M_{y y}^{0} \\
M_{x y}^{0}
\end{array}\right\}=\frac{\rho A^{2} h \alpha}{4} \omega^{2}\left\{\begin{array}{c}
N_{x x}^{0} \\
N_{y y}^{0} \\
N_{x y}^{0} \\
M_{x x}^{0} \\
M_{y y}^{0} \\
M_{x y}^{0}
\end{array}\right\}
$$

Recall that Eqs. (8.2) and (7.7) correspond to the element eigenvalue problem given by Eq. (2.7) with rigid body and hourglass modes neglected. As stated previously, $[m]$ is taken to be a lumped mass matrix. Although not shown here, it is well known that $[k]$ is real and symmetric. Hence, the matrix product $[m]^{-1}[k]$ is symmetric and real and therefore the eigenvalues of $[m]^{-1}[k]$ themselves are real. ${ }^{12}$ Obviously, this means that the eigenvalues of both $[P]$ and $\left[\tilde{A}_{t s}\right]$ are real. Using this fact along with the Gerschgorin circle theorem, a bound for the maximum membrane/bending frequency can be determined to be

$$
w_{\max } \leq \sqrt{\frac{4}{\rho A^{2} h \alpha} \max _{i}\left\{P_{i i}+r_{i}\right\}}
$$

where

$$
r_{i}=\sum_{\substack{j=1 \\ j \neq i}}^{n}\left|P_{i j}\right|
$$

Because $[P]$ is asymmetric, another bound can be developed by applying the Ger- 
schgorin Circle Theorem to the transpose of $[P]$ to give

$$
w_{\text {max }} \leq \sqrt{\frac{4}{\rho A^{2} h \alpha} \max _{j}\left\{P_{j j}+s_{j}\right\}}
$$

where

$$
s_{j}=\sum_{\substack{i=1 \\ i \neq j}}^{n}\left|P_{i j}\right|
$$

Because the two bounds given in Eqs. (8.3) and (8.5) are independent, the maximum allowable time step for membrane/bending waves is calculated using the minimum bound for the membrane/bending frequencies in Eq. (2.1). The critical time step corresponding to an element is then the minimum of those determined for membrane/bending and transverse shear waves. Finally, the critical time step for the entire system is bounded by the minimum critical time step over all of the elements in the mesh as stated in Eq. (2.3).

\section{$9 \quad$ Single Layer Reductions}

For a single layer, the $(6 \times 6)$ inplane eigenvalue problem decouples into independent $(3 \times 3)$ membrane and bending eigenvalue problems. The transverse shear eigenvalue problem remains decoupled and $(2 \times 2)$ in size. Closed-form expressions can be developed for the membrane, bending and transverse shear frequencies, but are only presented for the special sub-case where the material response is isotropic.

\subsection{General Single Layer}

Consider the single layer case where the constitutive behavior is described solely by Eqs. (6.1) and (6.2). Those equations either correspond to a truly anisotropic material with no coupling between inplane normal/shear and transverse shear behaviors or an

orthotropic material where the principal material directions are not aligned with the 
chosen $x-y-z$ system. In either case, the laminate matrices become

$$
\begin{aligned}
& {[A]=\left[\begin{array}{lll}
A_{11} & A_{12} & A_{16} \\
A_{12} & A_{22} & A_{26} \\
A_{16} & A_{26} & A_{66}
\end{array}\right]=h\left[\begin{array}{lll}
\bar{Q}_{11} & \bar{Q}_{12} & \bar{Q}_{16} \\
\bar{Q}_{12} & \bar{Q}_{22} & \bar{Q}_{26} \\
\bar{Q}_{16} & \bar{Q}_{26} & \bar{Q}_{66}
\end{array}\right]} \\
& {[B]=\left[\begin{array}{lll}
B_{11} & B_{12} & B_{16} \\
B_{12} & B_{22} & B_{26} \\
B_{16} & B_{26} & B_{66}
\end{array}\right]=\left[\begin{array}{ccc}
0 & 0 & 0 \\
0 & 0 & 0 \\
0 & 0 & 0
\end{array}\right]} \\
& {[D]=\left[\begin{array}{lll}
D_{11} & D_{12} & D_{16} \\
D_{12} & D_{22} & D_{26} \\
D_{16} & D_{26} & D_{66}
\end{array}\right]=\frac{h^{3}}{12}\left[\begin{array}{lll}
\bar{Q}_{11} & \bar{Q}_{12} & \bar{Q}_{16} \\
\bar{Q}_{12} & \bar{Q}_{22} & \bar{Q}_{26} \\
\bar{Q}_{16} & \bar{Q}_{26} & \bar{Q}_{66}
\end{array}\right]}
\end{aligned}
$$

and

$$
\left[A_{t s}\right]=\left[\begin{array}{ll}
A_{44} & A_{45} \\
A_{45} & A_{55}
\end{array}\right]=h\left[\begin{array}{ll}
\bar{Q}_{44} & \bar{Q}_{45} \\
\bar{Q}_{45} & \bar{Q}_{55}
\end{array}\right]
$$

The membrane and bending eigenvalue problems defined by Eq. (7.5) become

$$
\begin{gathered}
{[\tilde{Q}]\left\{\begin{array}{c}
N_{x x}^{0} \\
N_{y y}^{0} \\
N_{x y}^{0}
\end{array}\right\}=\frac{\rho A^{2}}{4} \omega_{m}^{2}\left\{\begin{array}{c}
N_{x x}^{0} \\
N_{y y}^{0} \\
N_{x y}^{0}
\end{array}\right\}} \\
{[\tilde{Q}]\left\{\begin{array}{c}
M_{x x}^{0} \\
M_{y y}^{0} \\
M_{x y}^{0}
\end{array}\right\}=\frac{3 \rho A^{2} \alpha}{h^{2}} \omega_{b}^{2}\left\{\begin{array}{c}
M_{x x}^{0} \\
M_{y y}^{0} \\
M_{x y}^{0}
\end{array}\right\}}
\end{gathered}
$$

where

$$
\begin{aligned}
{[\tilde{Q}] } & =\left[\begin{array}{ccc}
\tilde{Q}_{11} & \tilde{Q}_{12} & \tilde{Q}_{16} \\
\tilde{Q}_{21} & \tilde{Q}_{22} & \tilde{Q}_{26} \\
\tilde{Q}_{61} & \tilde{Q}_{62} & \tilde{Q}_{66}
\end{array}\right] \\
& =\left[\begin{array}{lll}
a_{11} \bar{Q}_{11}+a_{12} \bar{Q}_{16} & a_{22} \bar{Q}_{12}+a_{12} \bar{Q}_{16} & a_{12}\left(\bar{Q}_{11}+\bar{Q}_{12}\right)+\left(a_{11}+a_{22}\right) \bar{Q}_{16} \\
a_{11} \bar{Q}_{12}+a_{12} \bar{Q}_{26} & a_{22} \bar{Q}_{22}+a_{12} \bar{Q}_{26} & a_{12}\left(\bar{Q}_{12}+\bar{Q}_{22}\right)+\left(a_{11}+a_{22}\right) \bar{Q}_{26} \\
a_{11} \bar{Q}_{16}+a_{12} \bar{Q}_{66} & a_{22} \bar{Q}_{26}+a_{12} \bar{Q}_{66} & a_{12}\left(\bar{Q}_{16}+\bar{Q}_{26}\right)+\left(a_{11}+a_{22}\right) \bar{Q}_{66}
\end{array}\right]
\end{aligned}
$$

It should be clear that the decoupling of the membrane and bending eigenvalue problems occurs because $[B]$ is zero in this case. Furthermore, it should also be evident 
that the maximum bending frequency $\omega_{b_{\max }}$ is related to the maximum membrane frequency $\omega_{m_{\max }}$ as follows:

$$
\omega_{b_{\max }}=\left(\frac{h}{\sqrt{12 \alpha}}\right) \omega_{\max }
$$

If the rotational inertia scaling factor is chosen to be $h^{2} / 12$, then the membrane and bending eigenvalue problems are identical and the resulting maximum frequencies are equal. That is,

$$
\omega_{b_{\max }}=\omega_{\max } \quad \text { for } \alpha=\frac{h^{2}}{12}
$$

On the other hand, using $A / 12$ for the rotational inertia scaling gives

$$
\omega_{b_{\max }}=\left(\frac{h}{\sqrt{A}}\right) \omega_{\max } \quad \text { for } \alpha=\frac{A}{12}
$$

The transverse shear eigenvalue problem becomes

$$
\left[\tilde{Q}_{t s}\right]\left\{\begin{array}{c}
\beta_{y z} N_{y z}^{0} \\
\beta_{z x} N_{z x}^{0}
\end{array}\right\}=\frac{\rho A^{2} \alpha}{4} \omega_{t s}^{2}\left\{\begin{array}{c}
\beta_{y z} N_{y z}^{0} \\
\beta_{z x} N_{z x}^{0}
\end{array}\right\}
$$

where

$$
\left[\tilde{Q}_{t s}\right]=\left[\begin{array}{cc}
\tilde{Q}_{44} & \tilde{Q}_{45} \\
\tilde{Q}_{54} & \tilde{Q}_{55}
\end{array}\right]=\left[\begin{array}{c|c}
\left(\alpha a_{22}+a_{33}\right) \beta_{y z}^{2} \bar{Q}_{44} & \alpha \beta_{y z} \beta_{z x} a_{12} \bar{Q}_{44} \\
+\alpha \beta_{y z} \beta_{z x} a_{12} \bar{Q}_{45} & +\left(\alpha a_{11}+a_{33}\right) \beta_{z x}^{2} \bar{Q}_{45} \\
\hline\left(\alpha a_{22}+a_{33}\right) \beta_{y z}^{2} \bar{Q}_{45} & \alpha \beta_{y z} \beta_{z x} a_{12} \bar{Q}_{45} \\
+\alpha \beta_{y z} \beta_{z x} a_{12} \bar{Q}_{55} & +\left(\alpha a_{11}+a_{33}\right) \beta_{z x}^{2} \bar{Q}_{55}
\end{array}\right]
$$

\subsection{Single Specially Orthotropic Layer}

Here the term "specially orthotropic layer" is used to describe the case where an orthotropic layer has its principal material directions aligned with the chosen $x-y-z$ coordinate system. In such a case, the eigenvalue problems become simpler as

$$
\left[\begin{array}{ccc}
\bar{Q}_{11} & \bar{Q}_{12} & \bar{Q}_{16} \\
\bar{Q}_{12} & \bar{Q}_{22} & \bar{Q}_{26} \\
\bar{Q}_{16} & \bar{Q}_{26} & \bar{Q}_{66}
\end{array}\right]=\left[\begin{array}{ccc}
Q_{11} & Q_{12} & 0 \\
Q_{12} & Q_{22} & 0 \\
0 & 0 & Q_{66}
\end{array}\right]
$$

and

$$
\left[\begin{array}{ll}
\bar{Q}_{44} & \bar{Q}_{45} \\
\bar{Q}_{45} & \bar{Q}_{55}
\end{array}\right]=\left[\begin{array}{cc}
Q_{44} & 0 \\
0 & Q_{55}
\end{array}\right]
$$


The eigenvalue problems are once again defined by Eqs. (9.5), (9.6), and (9.11), but with $[\tilde{Q}]$ and $\left[\tilde{Q}_{t s}\right]$, respectively, simplified to be

$$
[\tilde{Q}]=\left[\begin{array}{ccc}
a_{11} Q_{11} & a_{22} Q_{12} & a_{12}\left(Q_{11}+Q_{12}\right) \\
a_{11} Q_{12} & a_{22} Q_{22} & a_{12}\left(Q_{12}+Q_{22}\right) \\
a_{12} Q_{66} & a_{12} Q_{66} & \left(a_{11}+a_{22}\right) Q_{66}
\end{array}\right]
$$

and

$$
\left[\tilde{Q}_{t s}\right]=\left[\begin{array}{cc}
\left(\alpha a_{22}+a_{33}\right) \beta_{y z}^{2} Q_{44} & \alpha \beta_{y z} \beta_{z x} a_{12} Q_{44} \\
\alpha \beta_{y z} \beta_{z x} a_{12} Q_{55} & \left(\alpha a_{11}+a_{33}\right) \beta_{z x}^{2} Q_{55}
\end{array}\right]
$$

\subsection{Single Isotropic Layer}

If the material response is isotropic, the closed-form expressions for the vibrational frequencies become manageable and will be presented herein.

\subsubsection{Membrane and Bending Behaviors}

First, however, it will be useful to explicitly write the reduced stiffnesses $\left(Q_{i j}\right.$ 's) in terms of $E$ and $\nu$ for the membrane and bending problems. The inplane isotropic elasticity tensor for the generalized plane stress case is given by

$$
\left[\begin{array}{ccc}
Q_{11} & Q_{12} & 0 \\
Q_{12} & Q_{22} & 0 \\
0 & 0 & Q_{66}
\end{array}\right]=\frac{E}{1-\nu^{2}}\left[\begin{array}{ccc}
1 & \nu & 0 \\
\nu & 1 & 0 \\
0 & 0 & \frac{1-\nu}{2}
\end{array}\right]
$$

The membrane vibrational frequencies are defined by the following eigenvalue problem

$$
\begin{aligned}
& {\left[\begin{array}{ccc}
a_{11} & \nu a_{22} & (1+\nu) a_{12} \\
\nu a_{11} & a_{22} & (1+\nu) a_{12} \\
\left(\frac{1-\nu}{2}\right) a_{12} & \left(\frac{1-\nu}{2}\right) a_{12} & \left(\frac{1-\nu}{2}\right)\left(a_{11}+a_{22}\right)
\end{array}\right]\left\{\begin{array}{c}
N_{x x}^{0} \\
N_{y y}^{0} \\
N_{x y}^{0}
\end{array}\right\}} \\
& =\frac{1-\nu^{2}}{E} \frac{\rho A^{2}}{4} \omega_{m}^{2}\left\{\begin{array}{c}
N_{x x}^{0} \\
N_{y y}^{0} \\
N_{x y}^{0}
\end{array}\right\}
\end{aligned}
$$


Once again, the bending eigenvalue problem differs only slightly and is given as

$$
\begin{aligned}
& {\left[\begin{array}{ccc}
a_{11} & \nu a_{22} & (1+\nu) a_{12} \\
\nu a_{11} & a_{22} & (1+\nu) a_{12} \\
\left(\frac{1-\nu}{2}\right) a_{12} & \left(\frac{1-\nu}{2}\right) a_{12} & \left(\frac{1-\nu}{2}\right)\left(a_{11}+a_{22}\right)
\end{array}\right]\left\{\begin{array}{c}
M_{x x}^{0} \\
M_{y y}^{0} \\
M_{x y}^{0}
\end{array}\right\}} \\
& =\frac{1-\nu^{2}}{E} \frac{3 \rho A^{2} \alpha}{h^{2}} \omega_{b}^{2}\left\{\begin{array}{c}
M_{x x}^{0} \\
M_{y y}^{0} \\
M_{x y}^{0}
\end{array}\right\}(9.1
\end{aligned}
$$

Because the maximum bending frequency is known in terms of the maximum membrane frequency via Eq. (9.8), only the membrane eigenvalue problem will be examined in detail. The vibrational frequencies associated with the three non-hourglass membrane modes are determined to be

$$
\left(\omega_{m}^{2}\right)_{1}=\frac{E}{1-\nu^{2}} \frac{2}{\rho A^{2}}\left(a_{11}+a_{22}\right)(1-\nu)
$$

and

$$
\left(\omega_{m}^{2}\right)_{2,3}=\frac{E}{1-\nu^{2}} \frac{2}{\rho A^{2}}\left(a_{11}+a_{22} \pm \sqrt{\left(a_{11}+a_{22}\right)^{2}-4\left(1-\nu^{2}\right)\left(a_{11} a_{22}-a_{12}^{2}\right)}\right)
$$

The maximum membrane frequency is then identified as

$$
\omega_{m_{\text {max }}}^{2}=\frac{E}{1-\nu^{2}} \frac{2}{\rho A^{2}}\left(a_{11}+a_{22}+\sqrt{\left(a_{11}+a_{22}\right)^{2}-4\left(1-\nu^{2}\right)\left(a_{11} a_{22}-a_{12}^{2}\right)}\right)
$$

An approximation which is often used involves bounding $\omega_{\text {max }}$ by disregarding the second term appearing in the square root. This is permissible as

$$
4\left(1-\nu^{2}\right)\left(a_{11} a_{22}-a_{12}^{2}\right)=\left(1-\nu^{2}\right)\left(y_{24}^{2} x_{31}^{2}+y_{31}^{2} x_{24}^{2}\right) \geq 0 \quad \forall \text { permissible } \nu
$$

Using this, the bound on $\omega_{m_{\max }}$ is given as follows:

$$
\omega_{m \max }^{2} \leq \frac{E}{1-\nu^{2}} \frac{4}{\rho A^{2}}\left(a_{11}+a_{22}\right)
$$

The exact critical time step associated with membrane waves is

$$
\left(\Delta t_{c r}\right)_{m}=\frac{2}{w_{m_{\max }}}
$$


Using Eq. (9.22), the exact $\left(\Delta t_{c r}\right)_{m}$ can be broken into the ratio of geometrical and material factors as follows:

$$
\left(\Delta t_{c r}\right)_{m}=\frac{\delta_{m}}{c_{m}}
$$

where $c_{m}$ is the plane stress membrane wave speed given by

$$
c_{m}=\sqrt{\frac{E}{\rho\left(1-\nu^{2}\right)}}
$$

and $\delta_{m}$ is the membrane characteristic length defined as

$$
\delta_{m}=\frac{A \sqrt{2}}{\sqrt{a_{11}+a_{22}+\sqrt{\left(a_{11}+a_{22}\right)^{2}-4\left(1-\nu^{2}\right)\left(a_{11} a_{22}-a_{12}^{2}\right)}}}
$$

The conservative bound on the membrane critical time step is determined from Eq. (9.24) to be

$$
\left(\Delta t_{c r}\right)_{m} \leq \frac{\left(\delta_{m}\right)_{a p p r o x}}{c_{m}}
$$

where

$$
\left(\delta_{m}\right)_{\text {approx }}=\frac{A}{\sqrt{a_{11}+a_{22}}}
$$

Using this bound eliminates several multiplications and an extra square-root operation for each element at each time step in a nonlinear analysis.

The bending critical time step is determined from that for membrane waves using Eq. (9.9) or (9.10) and is given, respectively, as

$$
\left(\Delta t_{c r}\right)_{b}=\left(\Delta t_{c r}\right)_{m} \quad \text { for } \alpha=\frac{h^{2}}{12}
$$

and

$$
\left(\Delta t_{c r}\right)_{b}=\frac{\sqrt{A}}{h}\left(\Delta t_{c r}\right)_{m} \quad \text { for } \alpha=\frac{A}{12}
$$

Note that it is quite possible that an element may exist in a mesh where $\sqrt{A}$ is smaller than $h$ such that $\left(\Delta t_{c r}\right)_{b}$ is smaller than $\left(\Delta t_{c r}\right)_{m}$ for the case where $\alpha$ is $A / 12$.

\subsubsection{Transverse Shear Behavior}

The elasticity tensor for the transverse shear response is given by

$$
\left[\begin{array}{cc}
Q_{44} & 0 \\
0 & Q_{55}
\end{array}\right]=\frac{E}{2(1+\nu)}\left[\begin{array}{ll}
1 & 0 \\
0 & 1
\end{array}\right]=G\left[\begin{array}{ll}
1 & 0 \\
0 & 1
\end{array}\right]
$$


The single isotropic layer transverse shear eigenvalue problem is thus given by

$$
\left[\begin{array}{cc}
\left(\alpha a_{22}+a_{33}\right) \beta_{y z}^{2} & \alpha \beta_{y z} \beta_{z x} a_{12} \\
\alpha \beta_{y z} \beta_{z x} a_{12} & \left(\alpha a_{11}+a_{33}\right) \beta_{z x}^{2}
\end{array}\right]\left\{\begin{array}{c}
\beta_{y z} N_{y z}^{0} \\
\beta_{z x} N_{z x}^{0}
\end{array}\right\}=\frac{1}{G} \frac{\rho A^{2} \alpha}{4} \omega_{t s}^{2}\left\{\begin{array}{c}
\beta_{y z} N_{y z}^{0} \\
\beta_{z x} N_{z x}^{0}
\end{array}\right\}
$$

The transverse shear vibrational frequencies are determined to be

$$
\begin{aligned}
\left(\omega_{t s}^{2}\right)_{1,2}= & \frac{2 G}{\rho A^{2} \alpha}\left(\left(\alpha a_{22}+a_{33}\right) \beta_{y z}^{2}+\left(\alpha a_{11}+a_{33}\right) \beta_{z x}^{2}\right) \\
& \pm \frac{2 G}{\rho A^{2} \alpha} \sqrt{\begin{array}{l}
\left.\left(\alpha a_{22}+a_{33}\right) \beta_{y z}^{2}+\left(\alpha a_{11}+a_{33}\right) \beta_{z x}^{2}\right)^{2} \\
-4 \beta_{y z}^{2} \beta_{z x}^{2}\left(\left(\alpha a_{22}+a_{33}\right)\left(\alpha a_{11}+a_{33}\right)-\alpha^{2} a_{12}^{2}\right)
\end{array}}
\end{aligned}
$$

Of course, the maximum of these two is

$$
\begin{aligned}
\omega_{t s_{\max }}^{2}= & \frac{2 G}{\rho A^{2} \alpha}\left(\left(\alpha a_{22}+a_{33}\right) \beta_{y z}^{2}+\left(\alpha a_{11}+a_{33}\right) \beta_{z x}^{2}\right) \\
& +\frac{2 G}{\rho A^{2} \alpha} \sqrt{\begin{array}{l}
\left.\left(\alpha a_{22}+a_{33}\right) \beta_{y z}^{2}+\left(\alpha a_{11}+a_{33}\right) \beta_{z x}^{2}\right)^{2} \\
-4 \beta_{y z}^{2} \beta_{z x}^{2}\left(\left(\alpha a_{22}+a_{33}\right)\left(\alpha a_{11}+a_{33}\right)-\alpha^{2} a_{12}^{2}\right)
\end{array}}
\end{aligned}
$$

Similar to the membrane and bending cases, a conservative bound on $\omega_{t s_{\max }}$ can be developed. First, the last term in the square root quantity is expanded as follows:

$$
\begin{aligned}
4 \beta_{y z}^{2} \beta_{z x}^{2}\left(\left(\alpha a_{22}+\right.\right. & \left.\left.a_{33}\right)\left(\alpha a_{11}+a_{33}\right)-\alpha^{2} a_{12}^{2}\right) \\
& =4 \beta_{y z}^{2} \beta_{z x}^{2}\left(\alpha^{2}\left(a_{11} a_{22}-a_{12}^{2}\right)+\alpha a_{33}\left(a_{11}+a_{22}\right)+a_{33}^{2}\right)
\end{aligned}
$$

Noting that each of the terms on the right hand side of Eq. (9.37) is positive, the following bound on $\omega_{t s_{\max }}$ results:

$$
\omega_{t s_{\max }} \leq \frac{2}{A} \sqrt{\frac{G}{\rho}} \sqrt{\left(a_{22}+\frac{a_{33}}{\alpha}\right) \beta_{y z}^{2}+\left(a_{11}+\frac{a_{33}}{\alpha}\right) \beta_{z x}^{2}}
$$

Replacing $\beta_{y z}$ and $\beta_{z x}$ by the maximum of these two quantities yields the following looser bound:

$$
\omega_{t s_{\max }} \leq \frac{2 \beta_{\max }}{A} \sqrt{\frac{G}{\rho}} \sqrt{a_{11}+a_{22}+\frac{2 a_{33}}{\alpha}}
$$

Similar to critical time steps for membrane and bending waves, the critical time step for transverse shear waves can be computed by dividing a characteristic element dimension by a wave speed. For the case where the exact formula for $\omega_{t s_{\max }}$ is used, the exact critical time step corresponding to transverse shear waves can be written as

$$
\left(\Delta t_{c r}\right)_{t s}=\frac{2}{\omega_{t s_{\max }}}=\frac{\delta_{t s}}{c_{t s}}
$$


where $c_{t s}$ is the transverse shear wave speed given as

$$
c_{t s}=\sqrt{\frac{G}{\rho}}
$$

and $\delta_{t s}$ is the characteristic length associated with transverse shear behavior defined as follows:

$$
\left(\delta_{c r}\right)_{t s}=\frac{A \sqrt{2 \alpha}}{\sqrt{\sqrt{\left(\alpha a_{22}+a_{33}\right) \beta_{y z}^{2}+\left(\alpha a_{11}+a_{33}\right) \beta_{z x}^{2}+}}}
$$

Similar to the membrane and bending cases, some computational effort can be saved by using conservative bounds on $\left(\Delta t_{c r}\right)_{t s}$. The bound corresponding to the frequency bound given in Eq. (9.38) is

$$
\left(\Delta t_{c r}\right)_{t s}=\frac{\left(\delta_{t s}\right)_{\text {approx-1 }}}{c_{t s}}
$$

where

$$
\left(\delta_{t s}\right)_{\text {approx }-1}=\frac{A}{\sqrt{\left(a_{22}+\frac{a_{33}}{\alpha}\right) \beta_{y z}^{2}+\left(a_{11}+\frac{a_{33}}{\alpha}\right) \beta_{z x}^{2}}}
$$

Likewise, the looser bound achieved by using the bound given in Eq. (9.39) is

$$
\left(\Delta t_{c r}\right)_{t s}=\frac{\left(\delta_{t s}\right)_{\text {approx-2 }}}{c_{t s}}
$$

where

$$
\left(\delta_{t s}\right)_{\text {approx }-2}=\frac{A}{\beta_{\max } \sqrt{a_{11}+a_{22}+\frac{2 a_{33}}{\alpha}}}
$$

Practically speaking, the additional computational expense of using Eq. (9.43) instead of Eq. (9.45) should be relatively small.

\subsubsection{Significance of Single Isotropic Layer Criteria}

The importance of computing the critical time step from the ratio of independent geometrical and material parameters in a CFL approach is that it allows quick estimates for $\Delta t_{c r}$ to be calculated even in the case of nonlinear material response. That is, in the case of a nonlinear constitutive equation, estimates for the current tangent moduli can be used in estimating wave speeds for use in the critical time step calculation. PRESTO typically uses this approach in such cases. 
Table 10.1: Nodal coordinates for single element example.

\begin{tabular}{cccc} 
Node & $x$ & $y$ & $z$ \\
& $($ in $)$ & $($ in $)$ & (in) \\
\hline \hline 1 & 0 & 0 & 0 \\
2 & $\frac{1}{\sqrt{2}}$ & $\frac{1}{\sqrt{2}}$ & 0 \\
3 & $\frac{1}{\sqrt{2}}$ & $2+\frac{1}{\sqrt{2}}$ & 0 \\
4 & 0 & 2 & 0 \\
\hline \hline
\end{tabular}

\section{Verification Problem}

Verification of the elastic_laminate stress evaluation has been accomplished through numerous regression tests. Several of these tests have been documented in Ref. 4. Here an example problem verifying the calculation of the critical time step will be described.

In this verification example, a single shell element has one node slightly perturbed in the $x$-direction. The actual deformation does not matter, because only the first critical time step calculation corresponding to the undeformed element geometry will be checked. The 4-layer laminate is composed of a contrived material system having the following engineering properties:

$$
\begin{aligned}
E_{1} & =26.25 \times 10^{6} \mathrm{psi} \\
E_{2} & =1.49 \times 10^{6} \mathrm{psi} \\
\nu_{12} & =0.28 \\
G_{12} & =1.04 \times 10^{6} \mathrm{psi} \\
G_{23} & =1.56 \times 10^{6} \mathrm{psi} \\
G_{31} & =2.6 \times 10^{6} \mathrm{psi}
\end{aligned}
$$

Each of the four layers is 0.01 in. thick. The laminate geometry is as shown in Fig. 10.1 with the nodal coordinates given in Table 10.1.

Relative to the global $x-y$ - $z$ coordinate system, the laminate is taken to have a stacking sequence of $[45 / 30 / 60 / 20]$, whereas relative to the element co-rotational $r$-s- $t$ coordinate system, the stacking sequence is $[0 /-15 / 15 /-25]$. Relative to the element 


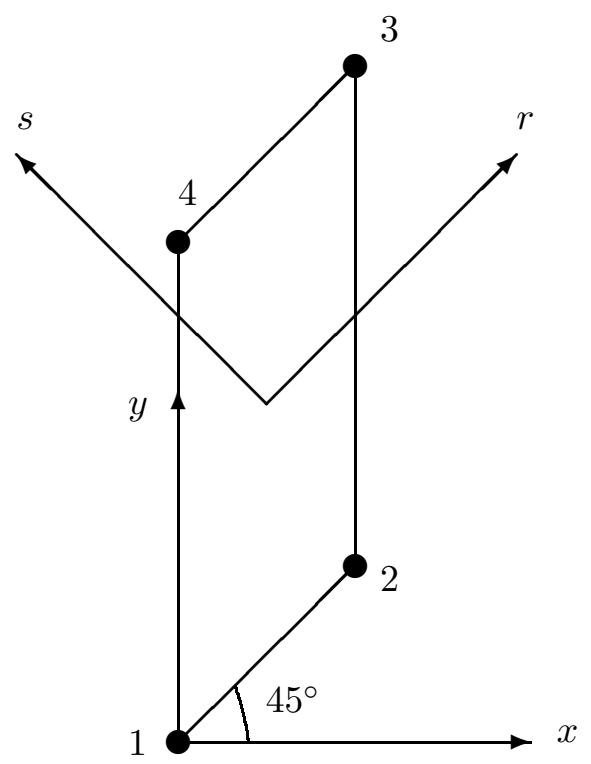

Figure 10.1: Example element geometry and co-rotational $r-s-t$ and global $x-y-z$ coordinate systems. The node numbers are also shown.

$r$-s-t coordinate system in which the material calculations are actually performed, the following laminate matrices result:

$$
\begin{aligned}
& {\left[\begin{array}{lll}
A_{11} & A_{12} & A_{16} \\
A_{12} & A_{22} & A_{26} \\
A_{16} & A_{26} & A_{66}
\end{array}\right]=\left[\begin{array}{ccc}
914.825 & 78.8905 & -75.7782 \\
78.8905 & 75.4794 & -19.482 \\
-75.7782 & -19.482 & 103.728
\end{array}\right] \times 10^{3} \mathrm{lb} / \mathrm{in}} \\
& {\left[\begin{array}{lll}
B_{11} & B_{12} & B_{16} \\
B_{12} & B_{22} & B_{26} \\
B_{16} & B_{26} & B_{66}
\end{array}\right]=\left[\begin{array}{ccc}
-1.16949 & 0.503184 & -0.578259 \\
0.503184 & 0.163124 & -0.228877 \\
-0.578259 & -0.228877 & 0.503184
\end{array}\right] \times 10^{3} \mathrm{lb}} \\
& {\left[\begin{array}{lll}
D_{11} & D_{12} & D_{16} \\
D_{12} & D_{22} & D_{26} \\
D_{16} & D_{26} & D_{66}
\end{array}\right]=\left[\begin{array}{ccc}
120.37 & 11.0151 & -17.6816 \\
11.0151 & 10.6776 & -4.5458 \\
-17.6816 & -4.5458 & 14.3267
\end{array}\right] \mathrm{lb} \cdot \mathrm{in}} \\
& {\left[\begin{array}{ll}
A_{44} & A_{45} \\
A_{45} & A_{55}
\end{array}\right]=\left[\begin{array}{ccc}
65.6508 & -3.98343 \\
-3.98343 & 100.749
\end{array}\right] \times 10^{3} \mathrm{lb} / \mathrm{in}}
\end{aligned}
$$

The chosen element geometry results in the following matrix of geometric factors for 
Table 10.2: Results for $\Delta t_{c r}$ for membrane/bending and transverse shear waves.

\begin{tabular}{cccc} 
Result & $\begin{array}{c}\text { Analytic-Exact } \\
(\mathrm{sec})\end{array}$ & $\begin{array}{c}\text { Analytic-Approximation } \\
(\mathrm{sec})\end{array}$ & $\begin{array}{c}\text { PRESTO } \\
(\mathrm{sec})\end{array}$ \\
\hline \hline$\Delta t_{c r}$ membrane/bending & 0.0075332 & 0.00642364 & 0.00642364 \\
$\Delta t_{c r}$ transverse shear & 0.151746 & - & 0.15174604 \\
\hline \hline
\end{tabular}

the $r$-s- $t$ system:

$$
\left[\begin{array}{lll}
a_{11} & a_{12} & a_{13} \\
a_{21} & a_{22} & a_{23} \\
a_{31} & a_{32} & a_{33}
\end{array}\right]=\left[\begin{array}{rrr}
2 & -2 & 0 \\
-2 & 3 & 0 \\
0 & 0 & 0.5
\end{array}\right] \mathrm{in}^{2}
$$

Note that the chosen geometry and stacking sequence results in all terms being present in the eigenvalue problems given in Eqs. (7.5) and (7.7).

The final results for the critical time steps corresponding to membrane/bending and transverse shear waves are shown in Table 10.2. Note that excellent agreement has been achieved between the PRESTO calculations and the appropriate analytic solutions. The exact analytic solutions are calculated directly from Eqs. (7.5), (7.7), and (2.1) and the approximate analytic solutions use the Gerschgorin circle bounds for membrane/bending waves given in Eqs. (8.3) and (8.5) along with Eq. (2.1). In Table 10.2, only the result from using Eq. (8.5) in Eq. (2.1) is shown as it allows for a larger acceptable time step than using Eq. (8.3) in Eq. (2.1). Here in this example, the final critical time step is that corresponding to membrane/bending waves, because it is smaller than that for transverse shear waves. 


\section{Summary}

The calculations required to estimate a critical time step for the elastic_laminate shell model which describes the small strain linear elastic response of laminated fiberreinforced composites have been detailed. By using a methodology very similar to that presented in Refs. 3 and 5, the eigenvalue problem for flat Mindlin shell elements has been reduced from finding the maximum frequency of a $(20 \times 20)$ system into two smaller eigenvalue problems corresponding to membrane/bending and transverse shear waves. The relevant membrane/bending frequency is found from the maximum eigenvalue of a $(6 \times 6)$ system, whereas the relevant transverse shear frequency is found from

a $(2 \times 2)$ system. Essentially, this reduction in problem size results from eliminating the rigid body modes and the hourglass modes resulting from single point integration at the element centroid. That is, the reduction comes from only considering the force and force-couple resultants that can be represented by such an under-integrated element. Reductions appropriate for a single layer laminate have been given. Furthermore, for the case of an isotropic layer, the resulting simplifications and approximations necessary to arrive at the formulas used in PRESTO have been detailed for the case where the effects of hourglass viscosity have been ignored.

In the PRESTO implementation of the critical time step estimates for the anisotropic elastic_laminate model, the maximum frequency corresponding to transverse shear waves is computed exactly under the assumptions used in this report, whereas the maximum frequency corresponding to membrane/bending waves is only estimated in order to minimize the computational cost. A verification problem from the PRESTO regression test suite has been detailed. 


\section{References}

[1] Bathe, K.J., Finite Element Procedures, Prentice-Hall, Englewood Cliffs, NJ, 1996.

[2] Cook, R., Malkus, D., Plesha, M. and Witt, R., Concepts and Applications of Finite Element Analysis, 4th ed., John Wiley \& Sons, Inc., New York, NY, 2002.

[3] Flanagan, D.P. and Belytschko, T., "Eigenvalues and Stable Time Steps for the Uniform Strain Hexahedron and Quadrilateral," Journal of Applied Mechanics, Vol. 51, 1984, pp. 35-40.

[4] Hammerand, D.C., Laminated Composites Modeling in ADAGIO/PRESTO, Sandia Report SAND2004-2143, Albuquerque, NM, 2004.

[5] Belytschko, T. and Lin, J.I., "Eigenvalues and Stable Time Steps for the Bilinear Mindlin Plate Element," International Journal for Numerical Methods in Engineering, Vol. 21, 1985, pp. 1729-1745.

[6] Krieg, R.D. and Key, S.W., "Transient Shell Response by Numerical Time Integration," International Journal for Numerical Methods in Engineering, Vol. 7, 1973, pp. 273-286.

[7] Hughes, T.J.R., Cohen, M. and Haroun, M., "Reduced and Selective Integration Techniques in Finite Element Analysis of Plates," Nuclear Engineering and Design, Vol. 46, 1978, pp. 203-222.

[8] Cowper, G.R., "On the Accuracy of Timoshenko's Beam Theory," Journal of the Engineering Mechanics Division, Proceedings of the American Society of Civil Engineers, Vol. 94, No. EM6, 1968, pp. 1447-1453.

[9] Fried, I., Johnson, A. and Tessler, A., "Minimal-Degree Thin Triangular Plate and Shell Bending Finite Elements of Order Two and Four," Computer Methods in Applied Mechanics and Engineering, Vol. 56, No. 3, 1986, pp. 283-307.

[10] Key, S.W., Petney, S.V. and Clancy, R.M., "The Impact Modeling of Structures Using Solid, Shell, and Membrane Finite Elements," Nuclear Engineering and Design, Vol. 116, No. 2, 1987, pp. 101-116. 
[11] Burden, R. and Faires, J., Numerical Analysis, 4th ed., PWS-Kent Publishing Company, Boston, MA, 1989.

[12] O'Neil, P., Advanced Engineering Mathematics, 3rd ed., Wadsworth Publishing Company, Belmont, CA, 1991. 


\section{Distribution}

$1 \quad$ MS 0139

P. Wilson, 9120

$1 \quad$ MS 0372

J. Jung, 9127

1 MS 0372

R. May, 9126

1 MS 0380

K. Alvin, 9142

1 MS 0380

M. Blanford, 9142

1 MS 0380

A. Gullerud, 9142

1 MS 0380 J. Hales, 9142

1 MS 0380 M. Heinstein, 9142

1 MS 0380

S. Key, 9142

1 MS 0380

R. Koteras, 9142

1 MS 0380

J. Mitchell, 9142

1 MS 0380

K. Pierson, 9142

1 MS 0380

V. Porter, 9142

1 MS 0380

G. Reese, 9142

1 MS 0384

T. Bickel, 9100

1 MS 0555

M. Garrett, 9122

1 MS 0557

T. Baca, 9125

1 MS 0615

D. Roach, 6252

1 MS 0847

H. Morgan, 9120

$1 \quad$ MS 0847

J. Redmond, 9124

$1 \quad$ MS 0847

H. Walther, 9127

1 MS 0888

D. Adolf, 1811

$1 \quad$ MS 0893

File Copy

1 MS 0893

R. Chambers, 9123

1 MS 0893

J. Cox, 9123

$1 \quad$ MS 0893

C. Lo, 9123

$20 \quad$ MS 0893

D. Hammerand, 9123

$1 \quad$ MS 0893

C. Lavin, 9123

1 MS 0893

M. Neilsen, 9123

1 MS 0893

J. Pott, 9123

MS 0893

E. Reedy, 9123

$1 \quad$ MS 0893

W. Scherzinger, 9123

MS 1110

D. Day, 9214

1 MS 9042

P. Spence, 8774

1 MS 9161

E. Chen, 8763

MS 9402 C. Cadden, 8772

1 MS 9403

J. Wang, 8773

1 MS 9405

K. Wilson, 8770

1 MS 9018

Central Technical Files, 8945-1

2 MS 0899 Technical Library, 9616 